\title{
SUBCELLULAR LOCALIZATION OF SARS-CoV STRUCTURAL PROTEINS
}

\author{
Lisa A. Lopez, Ariel Jones, William D. Arndt, and Brenda G. Hogue*
}

\section{INTRODUCTION}

Coronaviruses are enveloped viruses that assemble at intracellular membranes of the endoplasmic reticulum-Golgi intermediate compartment (ERGIC) in infected cells. ${ }^{1} \mathrm{~S}$ and $\mathrm{M}$ are the main components of the viral envelope. The $\mathrm{E}$ protein is a minor component of the envelope, but plays an important role in virus assembly. ${ }^{2}$ Coronavirus envelope formation is nucleocapsid independent. Expression of only the $E$ and $M$ proteins is sufficient for the formation of virus-like-particles (VLPs) of many coronaviruses. ${ }^{3-5}$ The $\mathrm{N}$ protein is a multifunctional phosphoprotein that encapsidates the viral genome and plays a role in virus assembly. ${ }^{6,7} \mathrm{~N}$ also appears to be involved in viral RNA replication and/or transcription. 8,9

The aim in this study was to provide a comprehensive view of the subcellular localization of the main SARS-CoV structural proteins. The S, M, E, and N genes were expressed in BHK-21 cells, and localization of the proteins was analyzed by indirect immunofluorescence microscopy. The proteins were co-analyzed with specific organelle markers for the endoplasmic reticulum (ER) and Golgi. Additionally, the $\mathrm{M}$ and $\mathrm{E}$ proteins were co-localized with a cellular marker for the ERGIC. The N protein remained cytoplasmic. The S, M, and E proteins were found to concentrate to the Golgi region, although some $\mathrm{S}$ appeared to also be transported to the cell surface.

\section{MATERIALS AND METHODS}

SARS-CoV M, E, and S genes were subcloned into the pCAGGS expression vector under the control of the chicken beta actin promoter. ${ }^{10}$ SARS-CoV $\mathrm{M}$ and $\mathrm{E}$ genes were cloned into pCAGGS with an HA tag on the amino terminus. BHK-21 cells were grown on Lab-Tek chamber slides (Nunc Inc.) and were transfected with pCAGGS DNAs using Lipofectamine (Invitrogen Life Technologies). Cells were fixed $18 \mathrm{~h}$ post-transfection in $100 \%$ methanol for $15 \mathrm{~min}$ at $-20^{\circ} \mathrm{C}$ and blocked overnight in PBS containing $0.2 \%$

*Arizona State University, Tempe, Arizona. 
gelatin. The $\mathrm{N}$ gene was cloned into a pcDNA vector (Invitrogen Life Technologies) under the control of the T7 promoter and expressed with vaccinia vTF7-3 that expresses T7 RNA polymerase. ${ }^{11}$ BHK-21 cells were infected with vTF7-3 $1 \mathrm{~h}$ prior to transfection of pcDNA-SARS-CoV N and fixed as described above at $3 \mathrm{~h}$ after transfection. Indirect immunofluorescence was done using primary antibodies $(\alpha-\mathrm{S}, \mathrm{CDC} ; \alpha-\mathrm{N}$, ViroStat; $\alpha$ HA, Santa Cruz; $\alpha$-Calnexin, StressGen; $\alpha$-Giantin, Convance; $\alpha$-ERGIC-53, Alexis Corp). Cells were dual labeled with secondary antibodies conjugated to FITC (Santa Cruz) or Alexa Fluor-594 (Molecular Probes). After washing, nuclei were stained with 4,6-diamidino-2-phenylindole (DAPI) and mounted with ProLong Gold Antifade reagent (Molecular Probes). Images were collected on an epifluorescence Nikon inverted microscope (Nikon Inc.) using MetaMorph imaging software (Universal Imaging Corporation). Images were processed using Adobe Photoshop.

\section{RESULTS AND DISCUSSION}

To determine the subcellular localization sites for SARS-CoV E, M, S, and N proteins, the genes were expressed in BHK-21 cells. Proteins were co-localized against the ER, ERGIC, and Golgi marker proteins, using calnexin, ERGIC-53, and giantin, respectively.

SARS-CoV M co-localized with the ERGIC/Golgi markers with no overlap with the ER marker calnexin (Fig. 1, upper left). There was no overlap between the $\mathrm{M}$ or $\mathrm{E}$ proteins and ER calnexin. Localization of the M protein in the Golgi is consistent with other coronaviruses. ${ }^{1}$ Our observation of $\mathrm{M}$ in the ERGIC agrees with a recent report which also noted an overlap of SARS-CoV M with the ERGIC in addition to localization in the Golgi. ${ }^{12}$

SARS-CoV E also localized to the Golgi (Fig. 1, upper right). Instead of a more punctuate pattern characteristic of ER, E exhibited a compact appearance that clearly overlapped the Golgi marker, giantin. Our lab has shown that mouse hepatitis A59 (MHV-CoV A59) E protein localizes in the perinuclear region that overlaps the ER (Lopez and Hogue unpublished data). Our results suggest that SARS and MHV E proteins localize differently. SARS-CoV E localization appears to be similar to avian infectious bronchitis virus (IBV) E, which localizes to the Golgi. ${ }^{5}$

SARS-CoV S protein co-localized with the ER and Golgi markers and was detected in compartments along the secretory pathway (Fig. 1, lower panel). This is typical of proteins that are transported to the cell surface. However the SARS-CoV S protein appeared to concentrate primarily in the Golgi region. The focused localization of S when expressed alone suggests there may be some functional significance for the presence of the protein near the site of SARS-CoV budding and assembly. Our results are consistent with the recent report demonstrating that SARS-CoV S contains a novel dibasic motif that retains the protein in the ERGIC. ${ }^{13}$

The $\mathrm{N}$ protein remained in the cytoplasm (Fig. 1, middle panel). The protein did not co-localize with either the ER or Golgi markers. Other coronavirus N proteins have been localized to both the cytoplasm and the nucleolus. ${ }^{14}$ Several reports indicate that SARS$\mathrm{CoV} \mathrm{N}$ is transported to the nucleus, whereas another did not observe nuclear localization. ${ }^{15-18}$ Consistent with the latter, we were unable to detect SARS-CoV N in the 
nucleus. More comprehensive studies are clearly required to fully resolve and understand the trafficking of the $\mathrm{N}$ protein.

Our data show that while there are obvious similarities in the localization of SARS$\mathrm{CoV}$ structural proteins and those of other coronaviruses, there are apparent differences. Ultimately, it will be important to compare our data with staining patterns seen in SARS$\mathrm{CoV}$ infected cells to get a complete picture of where these structural proteins localize in the context of the other viral proteins. Understanding viral protein trafficking and identifying which protein(s) directs the site of virus assembly will help us develop antiviral drug platforms and possible vaccines to combat this important pathogen.
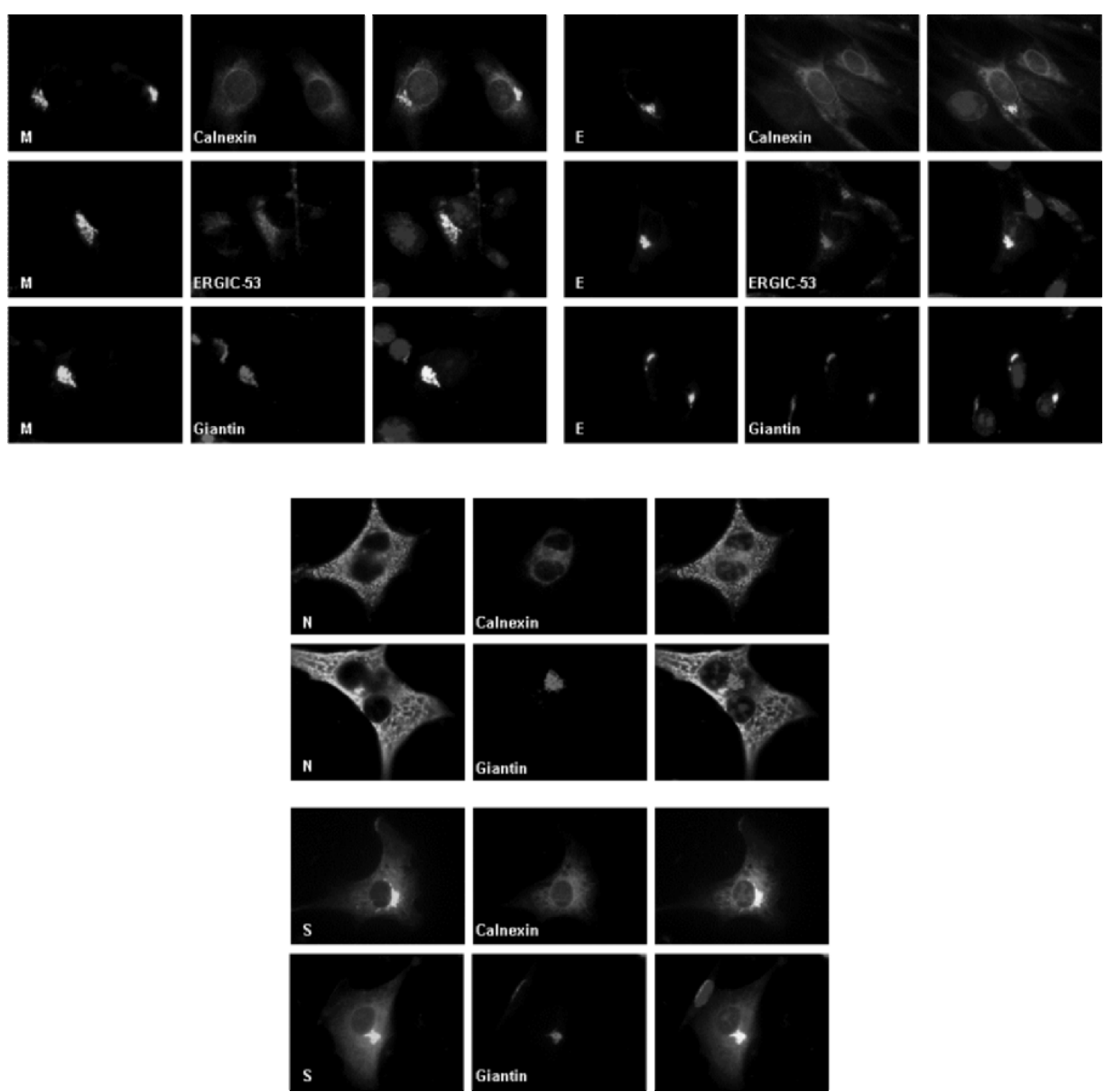

Figure 1. Subcellular localization of HA-tagged SARS-CoV M and E proteins, and localization of $\mathrm{N}$ and $\mathrm{S}$ proteins transiently expressed in BHK-21 cells. Singly expressed M, E, N, and S proteins were co-localized with cellular marker proteins. Cells were fixed and dual labeled for viral proteins and the corresponding marker proteins. Merged images are shown in the far right column $(100 \times$ magnification). 


\section{ACKNOWLEDGMENTS}

This work was supported by Public Health Service grant AI54704 from the National Institute of Allergy and Infectious Diseases. We thank Jarrod Lauer and Tiana White for help in generating the $\mathrm{M}, \mathrm{E}$, and $\mathrm{N}$ subclones.

\section{REFERENCES}

1. K. V. Holmes and M. M. Lai, in: Fields Virology, $4^{\text {th }}$ edition, edited by B. N. Fields, D. N. Knipe, and P. M. Howley (Lippincott-Raven Publishers, Philadelphia, 2001).

2. L. Kuo and P. S. Masters, The small envelope protein $\mathrm{E}$ is not essential for murine coronavirus replication, J. Virol. 77, 4597-4608 (2003).

3. H. Vennema, G. J. Godeke, J. W. Rossen, W. F. Voorhout, M. C. Horzinek, D. J. Opstelten, and P. J. Rottier, Nucleocapsid-independent assembly of coronavirus-like particles by co-expression of viral envelope protein genes, EMBO J. 15, 2020-2028 (2000).

4. P. Baudoux, C. Carrat, L. Besnardeau, B. Charley, and H. Laude, Coronavirus pseudoparticles formed with recombinant $\mathrm{M}$ and $\mathrm{E}$ proteins induce alpha interferon synthesis by leukocytes, J. Virol. 72, 8636-8643 (1998).

5. E. Corse and C. E. Machamer, Infectious bronchitis virus E protein is targeted to the Golgi complex and directs release of virus-like particles, J. Virol. 74, 4319-4326 (2000).

6. D. Escors, J. Ortego, H. Laude, and L. Enjuanes, The membrane M protein carboxy terminus binds to transmissible gastroenteritis coronavirus core and contributes to core stability, J. Virol. 75, 1312-1324 (2001).

7. K. Narayanan, K. H. Kim, and S. Makino, Characterization of N protein self-associations in coronavirus ribonucleoprotein complexes, Virus Res. 98, 131-140 (2003).

8. F. Almazan, C. Galan, and L. Enjuanes, The nucleoprotein is required for efficient coronavirus genome replication, J. Virol. 78, 12683-12688 (2004).

9. B. Schelle, N. Karl, B. Ludewig, S. G. Siddell, and V. Thiel, Selective replication of coronavirus genomes that express nucleocapsid protein, J. Virol. 79, 6620-6630 (2005).

10. H. Niwa, K. Yamamura, and J. Miyazaki, Efficient selection for high-expression transfectants with a novel eukaryotic vector, Gene 108, 193-199 (1991).

11. T. R. Fuerst, E. G. Niles, F. W. Studier, and B. Moss, Eukaryotic transient-expression system based on recombinant vaccinia virus that synthesizes bacteriophage T7 RNA polymerase, Proc. Natl. Acad. Sci. USA 83, 8122-8126 (1986).

12. B. Nal, C. Chan, F. Kien, L. Siu, J. Tse, K. Chu, J. Kam, I. Staropoli, B. Crescenzo-Chaigne, N. Escriou, S. vander Werf, K.-Y. Yuen, and R. Altmeyer, Differential maturation and subcellular localization of severe acute respiratory syndrome coronavirus surface proteins S, M and E, J. Gen. Virol. 86, 1423-1434 (2005).

13. E. Lontok, E. Corse, and C. E. Machamer, Intracellular targeting signals contribute to localization of coronavirus spike proteins near the virus assembly site, J. Virol. 78, 5913-5922 (2004).

14. T. Wurm, H. Chen, T. Hodgson, P. Britton, G. Brooks, and J. A. Hiscox, Localization to the nucleolus is a common feature of coronavirus nucleoproteins, and the protein may disrupt host cell division, J. Virol. 75 , 9345-9356 (2001).

15. M. A. Chang, Y. T. Lu, S. T. Ho, C. C, Wu, T. Y. Wei, C. J. Chen, Y. T. Hsu, P. C. Chu, C. H. Chen, J. M. Chu, Y. L. Jan, C. C. Hung, C. C. Fan, and Y. C. Yang, Antibody detection of SARS-CoV spike and nucleocapsid protein, Biochem. Biophys. Res. Commun. 314, 931-936 (2004).

16. R. Zeng, H. Q. Ruan, X. S. Jiang, H. Zhou, L. Shi, L. Zhang, Q. H. Sheng, Q. Tu, Q. C. Xia, and J. R. Wu, Proteomic analysis of SARS associated coronavirus using two-dimensional liquid chromatography mass spectrometry and one-dimensional sodium dodecyl sulfate-polyacrylamide gel electrophoresis followed by mass spectrometric analysis, J. Proteome Res. 3, 549-555 (2004).

17. M. Surjit, R. Kumar, R. N. Mishra, M. K. Reddy, V. T. Chow, and S. K. Lal, The severe acute respiratory syndrome coronavirus nucleocapsid protein is phosphorylated and localizes in the cytoplasm by 14-3-3mediated translocation, J. Virol. 79, 11476-11486 (2005).

18. R. R. Rowland, V. Chauhan, Y. Fang, A. Pekosz, M. Kerrigan, and M. D. Burton, Intracellular localization of the severe acute respiratory syndrome coronavirus nucleocapsid protein: absence of nucleolar accumulation during infection and after expression as a recombinant protein in Vero cells, J. Virol. 79, 11507-11512 (2005). 